\title{
The Impact of Spiritual Wellbeing and Resiliency in Predicting Death Anxiety among Elderly People in
}

Tehran

\author{
Abdullah Moetamedi ${ }^{1}$, Shima Pajouhinia ${ }^{*}$, Seyed Mohammad Hasan Fatemi Ardestani ${ }^{2}$ \\ ${ }^{1}$ Psychology Department, Education and Psychology Faculty, Allameh Tabatabaei University, Tehran, Iran \\ ${ }^{2}$ Department of Qur'an and Hadith Sciences, Usul Al Deen College, Tehran, Iran
}

\section{A BSTRACT}

Introduction: The Near-death experience is one of the undeniable facts of aging. This study was aimed to examine the role of spiritual wellbeing and resiliency in predicting death anxiety among elderly people in Tehran, Iran. Materials and Methods: The method of this study was descriptive-correlational with the total number of 200 older males who live in Tehran during 2013-2014. The questionnaires of the Spiritual Wellbeing Scale, Resiliency Scale, and Death Anxiety Scale were used for this investigation. Results: Our findings revealed that there was a negative significant correlation between spiritual wellbeing and resiliency with death anxiety. In addition, the results showed that spiritual wellbeing was a valuable factor in prediction of death anxiety. Conclusion: Our data indicated that resiliency and spiritual wellbeing can be considered as crucial factors in death anxiety among the older adults.

* Corresponding Author: Shima Pajouhinia

E-mail: sh.pajouhinia@yahoo.com 
تأثير بهزيستى معنوى و تابآورى در ييشبينى اضطراب مرى در ميان سالمندان در شهر تهران

\author{
عبدالله معتمدى'، شيما يزوهى نيا'، سيدمحمدحسن فاطمى اردستانى' \\ 'كروه روانشناسى، دانشكده علوم تربيتى و روانشناسى، دانشكاه علامه طباطبائى، تهران، ايران \\ rكروه علوم قرآن و حديث، دانشكده اصول الدين، تهران، ايران
}

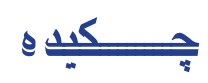

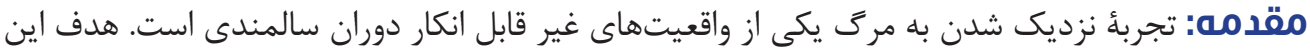

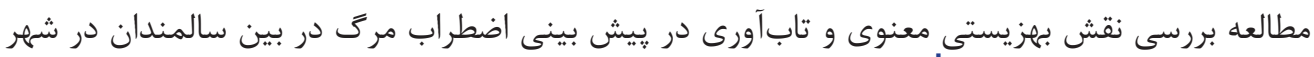

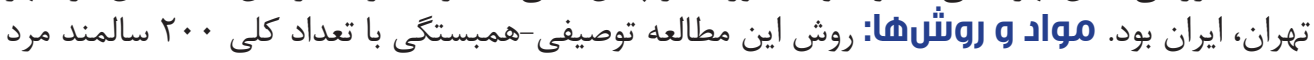

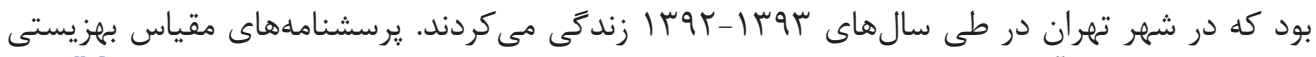

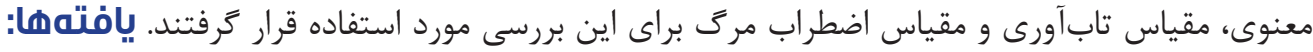

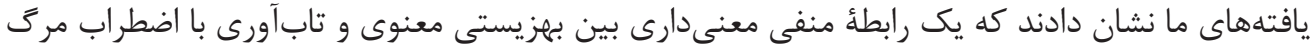

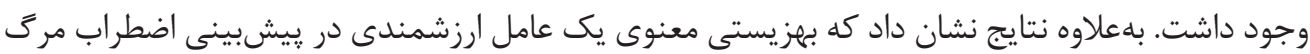

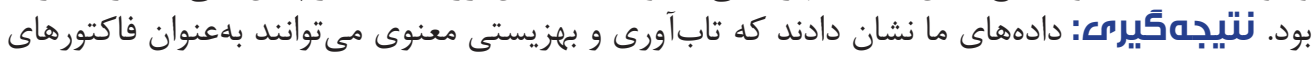

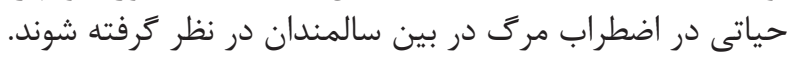

كليد وازهها:

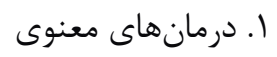

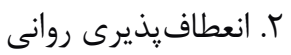

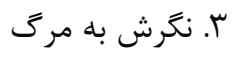

" نويسنده مسئول: شيما بزوهى نيا آدرس الكترونيكى: sh.pajouhinia@yahoo.com 
از مرگ و مردن ممكن است عمومى باشد اما افراد در ابراز

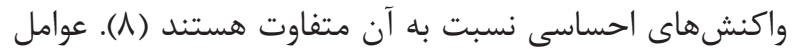

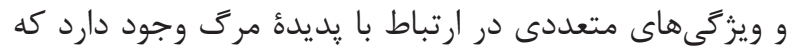

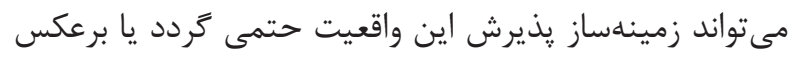

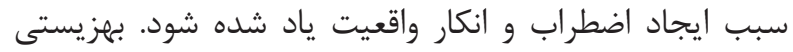

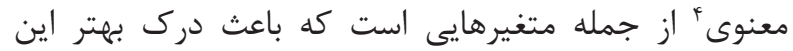

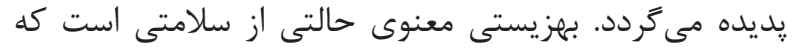

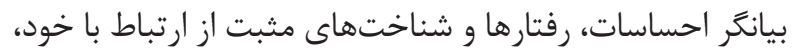

ديخران، طبيعت و موجودى برتر مىباشد (9) (9).

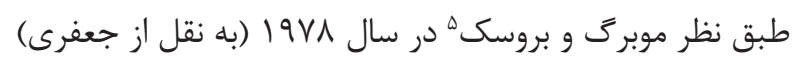

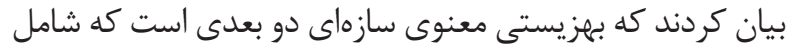

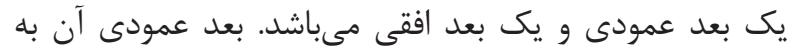

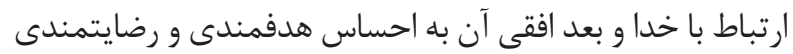

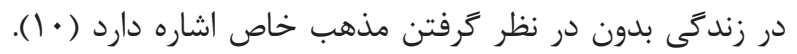

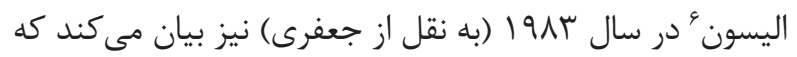

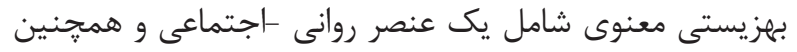

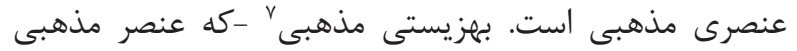

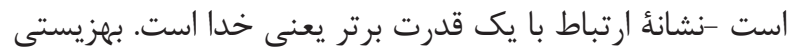

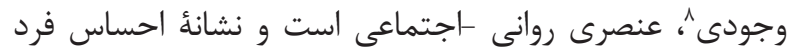

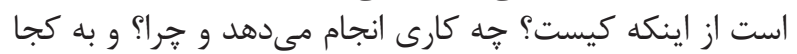

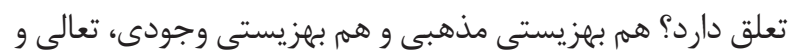

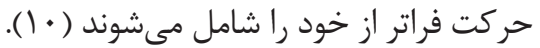

يزوهشهاى زيادى نشان داده است كه بهزيستى معنوى،

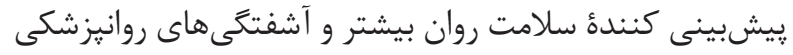

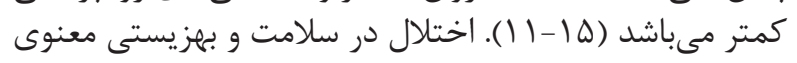

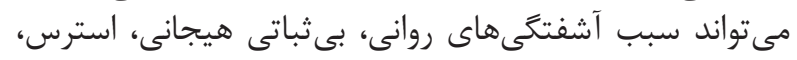

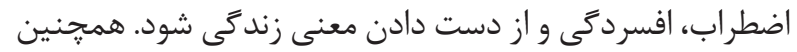

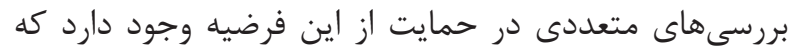

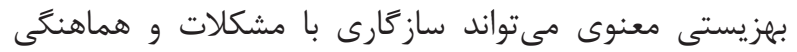

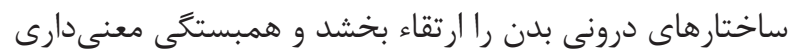

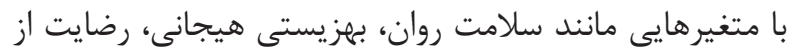

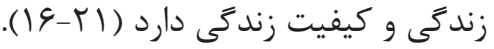

كموبيش صاحبنظران معتقدند كه باورها و معنويت در ميان

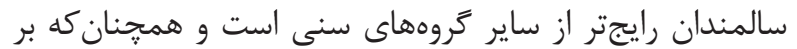

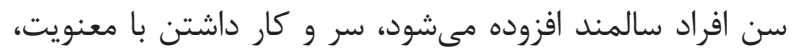

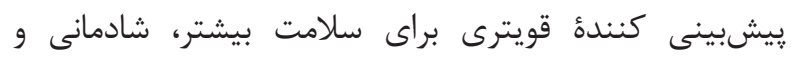

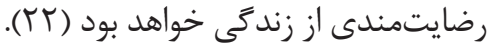

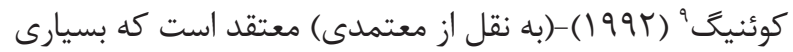

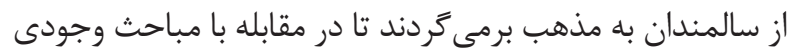

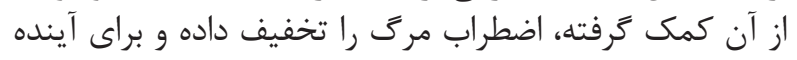

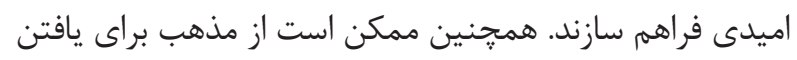

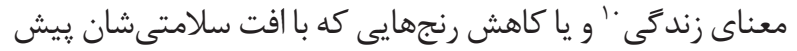

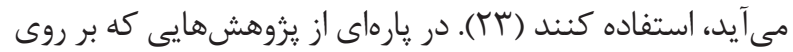

${ }^{1}$ Ageing

${ }^{2}$ Death anxiety

${ }^{3}$ Belsky

${ }^{4}$ Spiritual wellbeing

${ }^{5}$ Moberg and Brusek

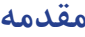

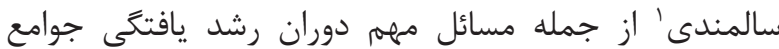

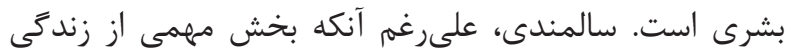

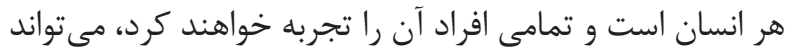

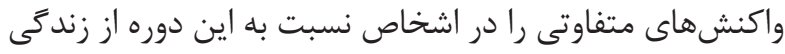

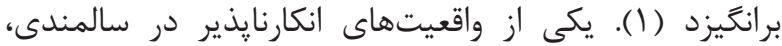

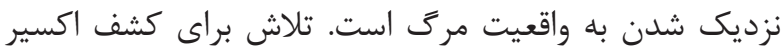

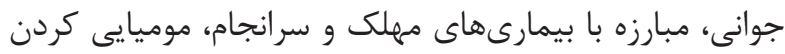

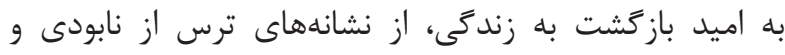

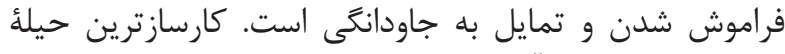

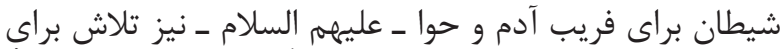

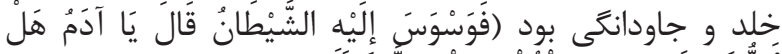

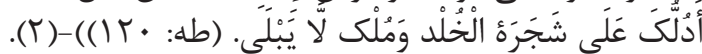

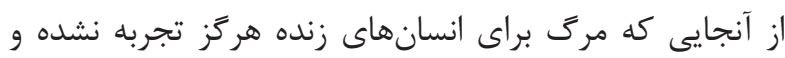

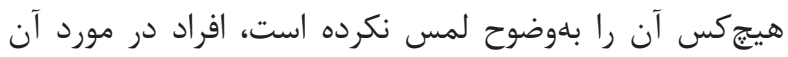

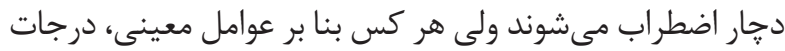

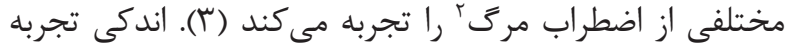

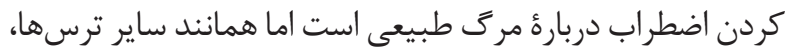

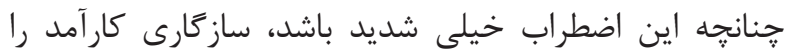

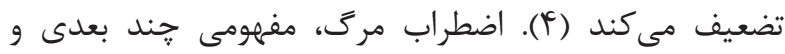

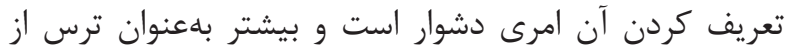

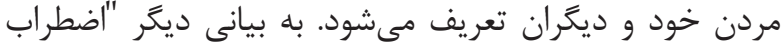

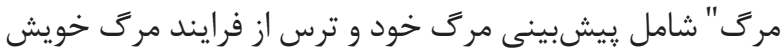

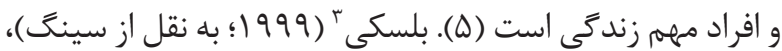

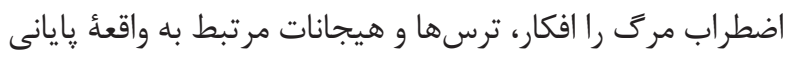

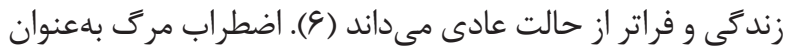

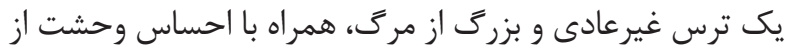

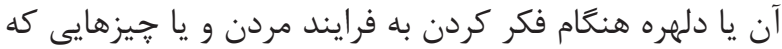

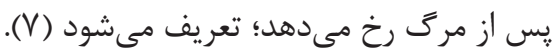

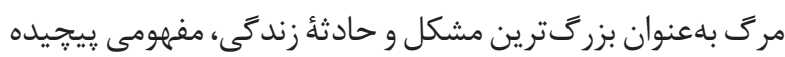

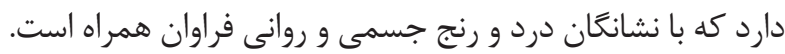

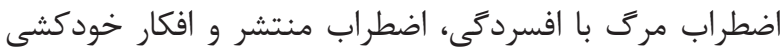

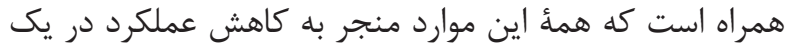

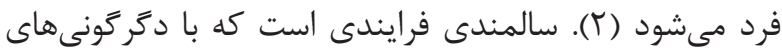

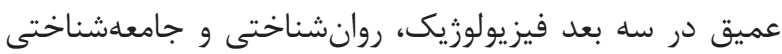

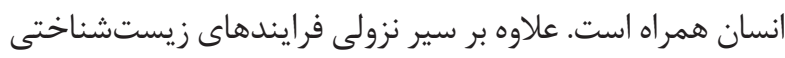

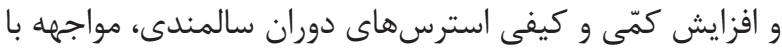

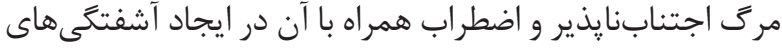
روانى اين دوره نقش مهمى دارئن إنداب

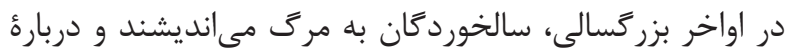

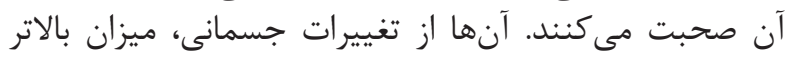

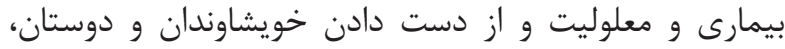

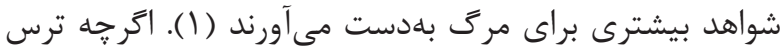

\footnotetext{
${ }^{6}$ Ellison

${ }^{7}$ Religious wellbeing

${ }^{8}$ Existential wellbeing

${ }^{9}$ Koenig

${ }^{10}$ The meaning of life
} 
سالمندان شناسايى، اندازمخيرى و كنترل شود تا در نتيجه به به كاهش اضطراب مرك در زندَّى سالمندان كمك نمايد.

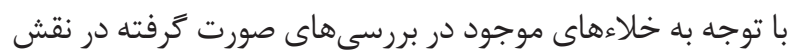

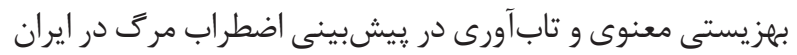

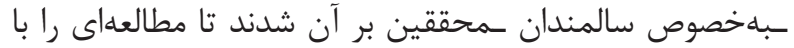

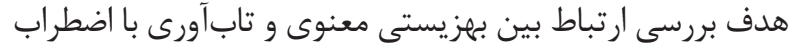

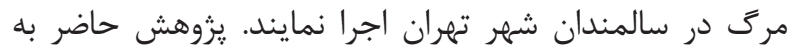

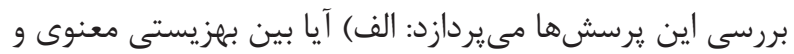

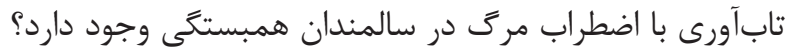

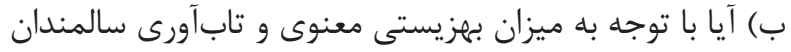

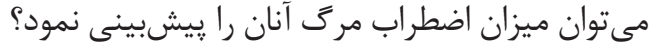

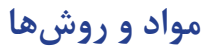

روش يزؤهش، جامعلُ آمارى و نمونه: تحقيق حاضر از نوع

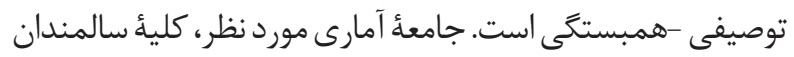

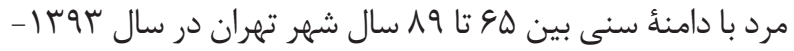

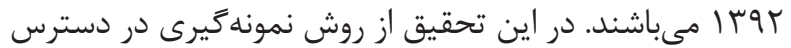

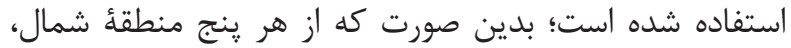

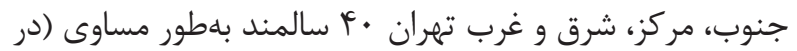

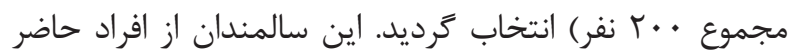

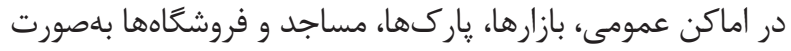

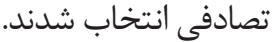

ابزار سنجش - ابش

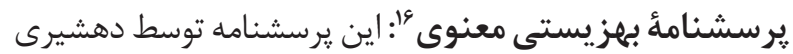

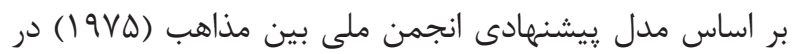

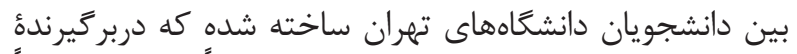

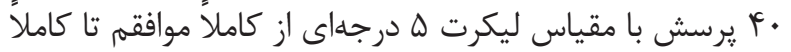

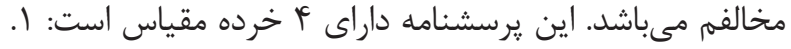

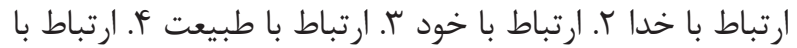

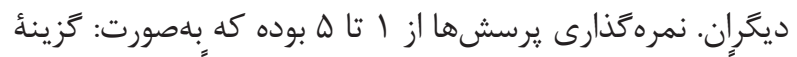

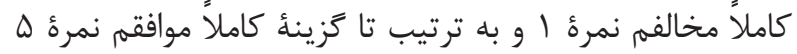

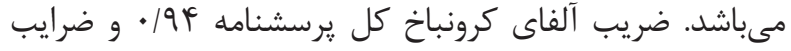

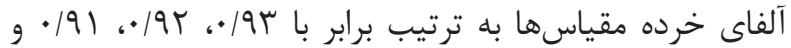

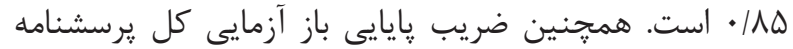

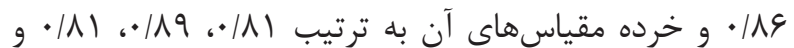

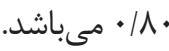

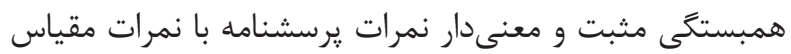

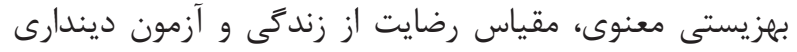

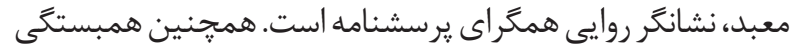

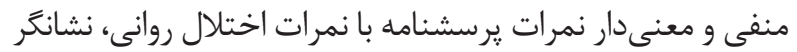

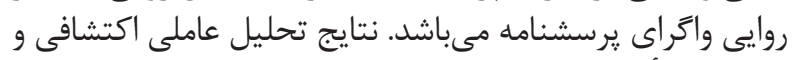

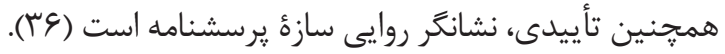

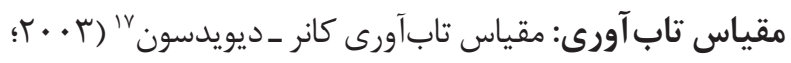

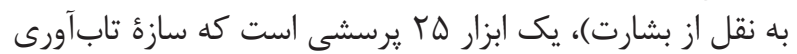

${ }^{11}$ Fortner and Neimeyer

${ }^{12}$ Resiliency

${ }^{13}$ Strategies

${ }^{14}$ Ong

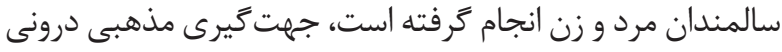
به صورت منفى و جهت

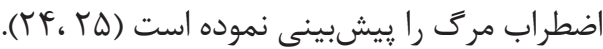
نكَرشهاى معنوى روش برخورد افراد با مرك، ديدَّاه و درى

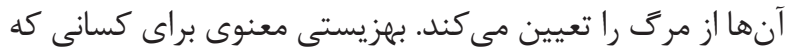

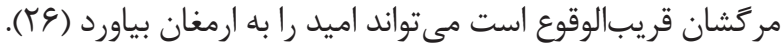

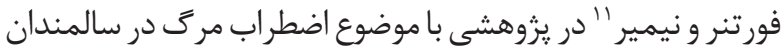

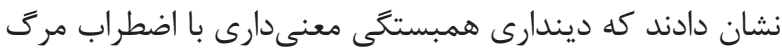

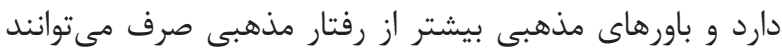

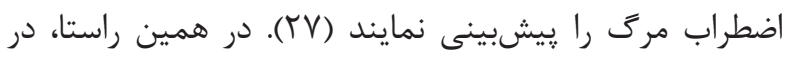

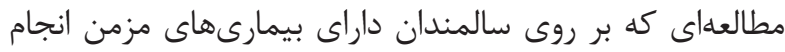

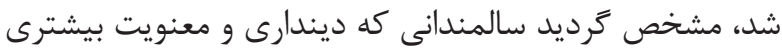

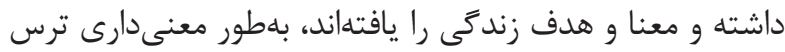

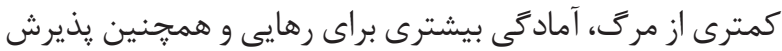

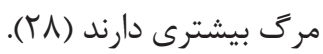

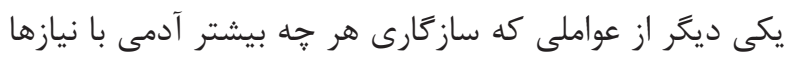

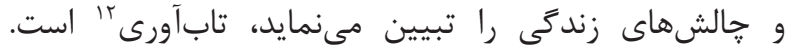

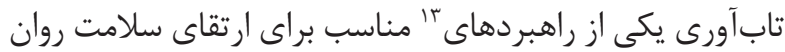

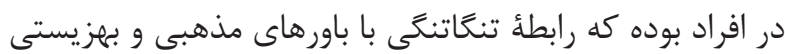

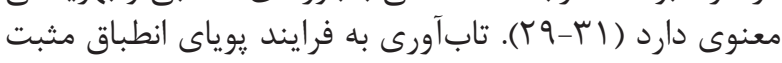

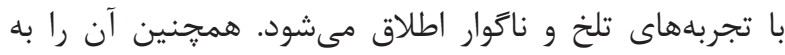

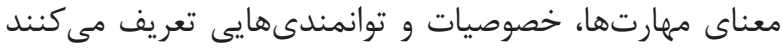

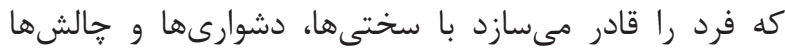

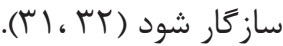

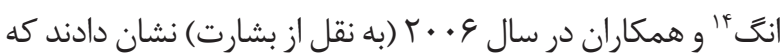

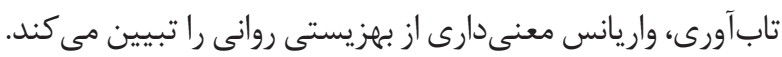

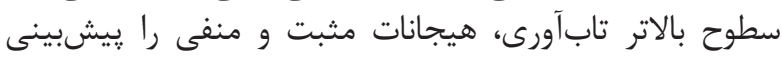

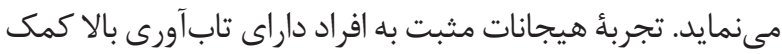

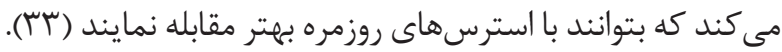

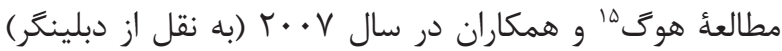

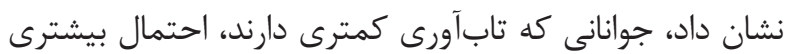

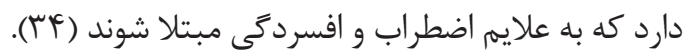

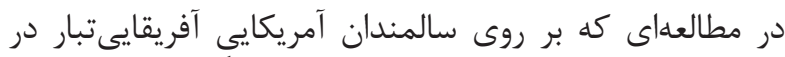

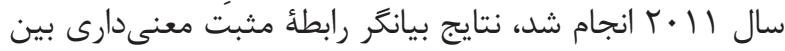

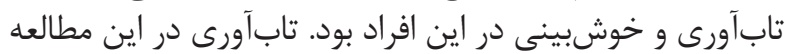

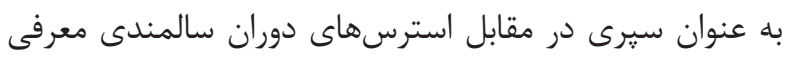

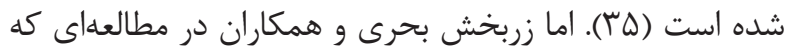

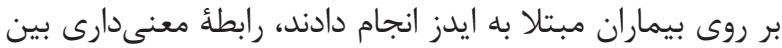
تابآورى و اضطراب مرى نيافتند (V)

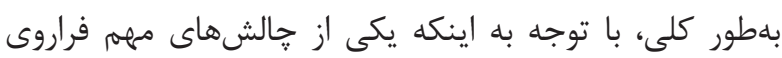

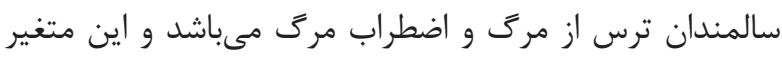

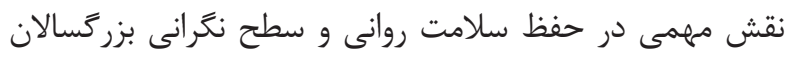

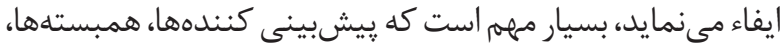

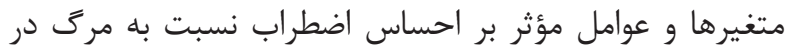

${ }^{15} \mathrm{Hoge}$

${ }^{16}$ Spiritual Wellbeing Scale (SWBS)

${ }^{17}$ Connor-Davidson, Resilience Scale (CD-RISC) 


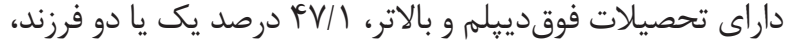

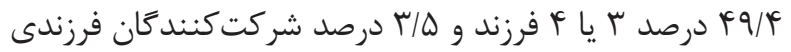

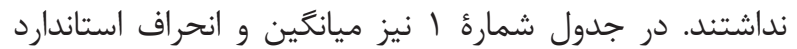
متغيرهاى يزوهش در تروه نمونه بيان شده است.

\begin{tabular}{|c|c|c|}
\hline انحراف استاندارد & ميانگين & متغيرها \\
\hline$I V / I T$ & $\mid 9 \mathrm{~V} / \cdot \mathrm{V}$ & بهزيستى معنوى \\
\hline $11 / \cdot r$ & VT/GD & تابآورى \\
\hline$\because a^{1 / \pi q}$ & $9(0)$ & اضطراب مرى \\
\hline
\end{tabular}

در جدول ك، با توجه به سطح معنى دارى بهدست آمده، مىتوان

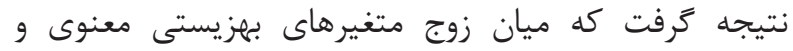

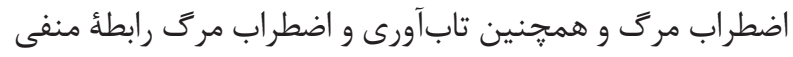

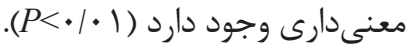

جدول r- نتايج تحليل همبستىى متغيرهاى مستقل با اضطراب مركى.

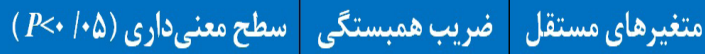

\begin{tabular}{|c|c|c|}
\hline .1 .1 & $-\cdot 1 / 19$ & بهز يستى معنوى \\
\hline .1 .1 &.$- \pi \omega$ & تابآورى \\
\hline
\end{tabular}

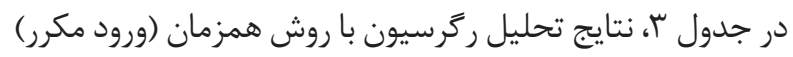

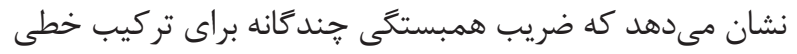

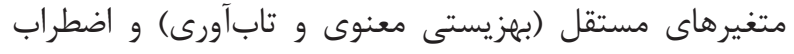

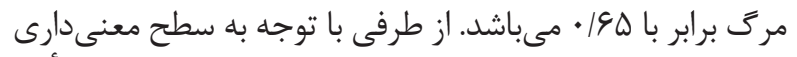

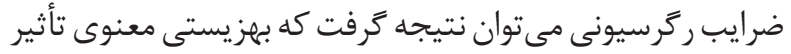

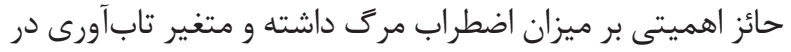

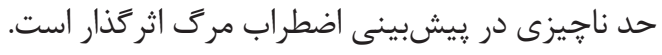

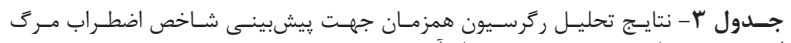
از روى متغيرهاى بجزيستى معنوى و تاب آورى.

\begin{tabular}{|c|c|c|c|c|c|c|}
\hline \multirow{2}{*}{ ضريب تعيين (R2) } & \multirow{2}{*}{ معنى إرى } & \multirow{2}{*}{ آمارة t } & \multirow{2}{*}{ ضرايب استاندارن } & \multicolumn{2}{|c|}{ ضرايب غير استاندارد } & \multirow{2}{*}{ شاخصنهائ مدل } \\
\hline & & & & انحراف معيار & B & \\
\hline \multirow{3}{*}{.190} & $\cdot 1$ & kr/af & - & $\cdot \lambda r$ & 19:DV & ضريب ثابت \\
\hline & .1 & $-|V| 19$ & -.190 & .199 & $-k \pi r$ & بيزيستى معنوى \\
\hline & $\| \lambda$ & $-1, \pi r$ & $-\pi \mu^{4}$ & $\| \mu^{4}$ & $-\pi, \pi \mu$ & تابآرث \\
\hline
\end{tabular}

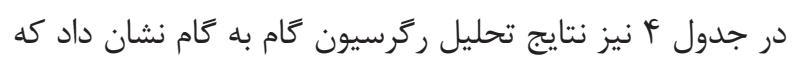

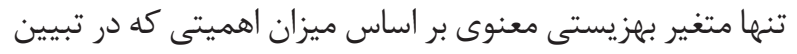

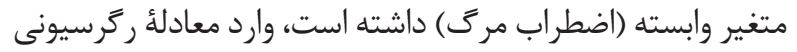

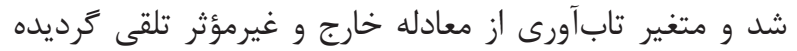

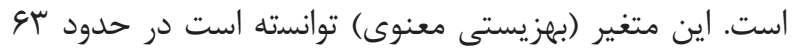

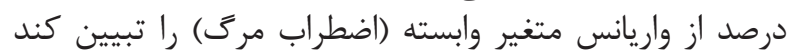
. $\left(\mathrm{R}^{2}=\cdot 19 \boldsymbol{H}^{\mathrm{T}}\right)$
را در اندازههاى ينجدرجهاى ليكرت از صفر تا هجهار مىسنجد.

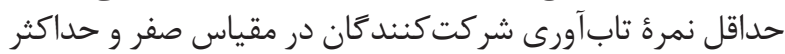

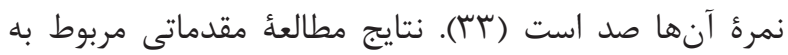

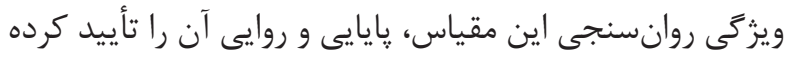

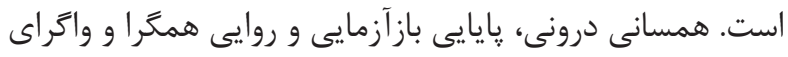

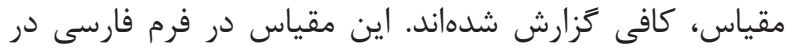

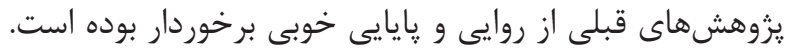

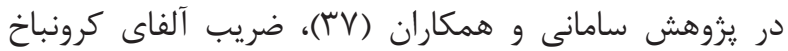

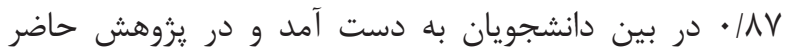

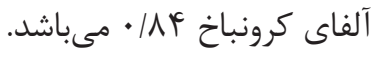

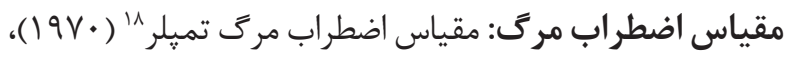

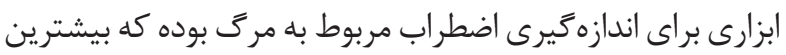

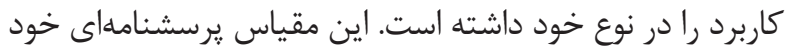

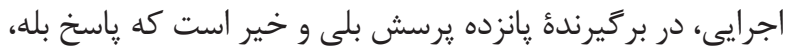

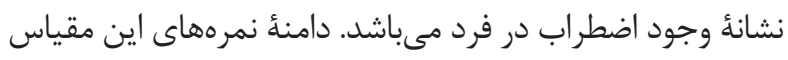

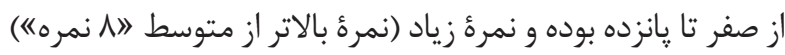

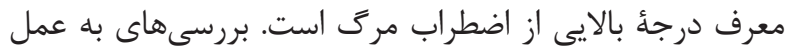

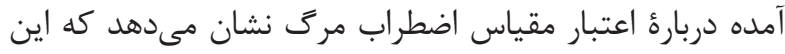

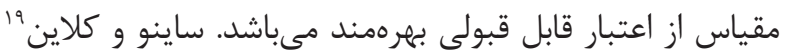

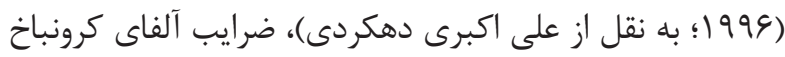

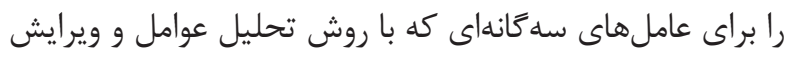

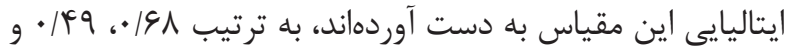

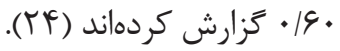

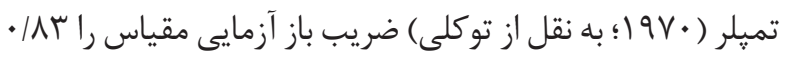

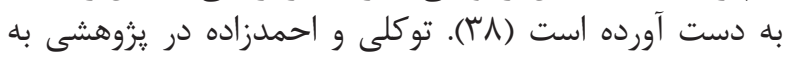

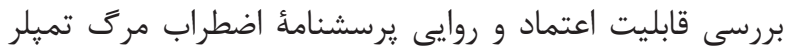

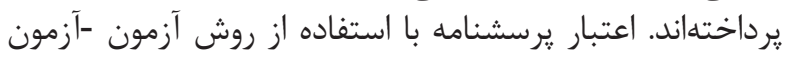

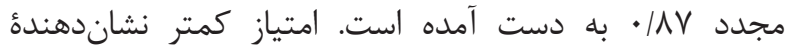

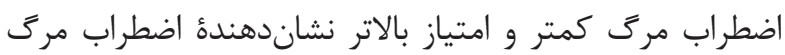

بيشتر در سالمندان است (^یّ).

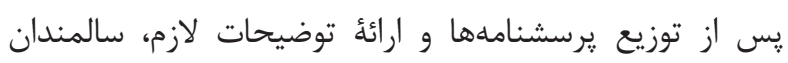

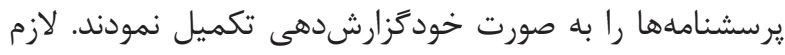

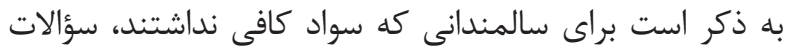

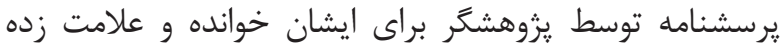

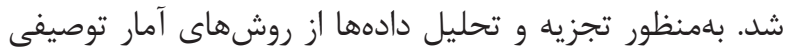

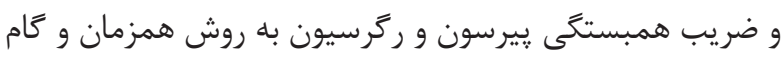

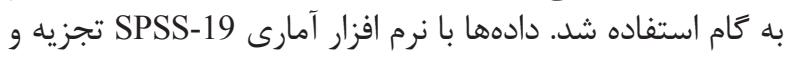

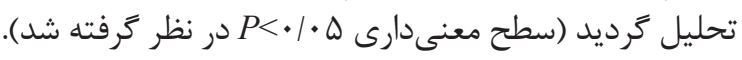

بافتهها

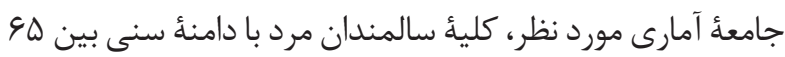

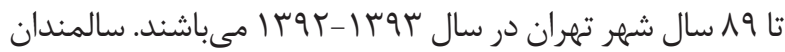

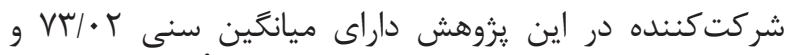

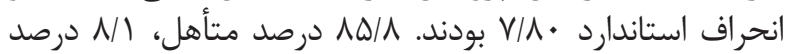

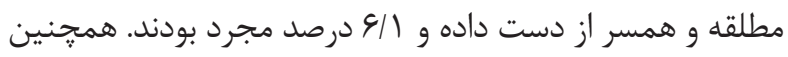

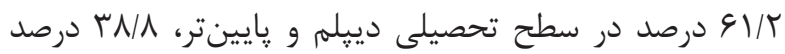

${ }^{18}$ Templer, Death Anxiety Scale

${ }^{19}$ Saggino and Kline 


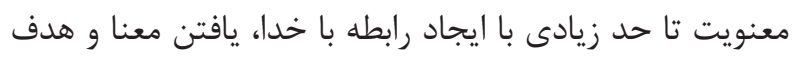

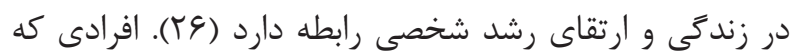

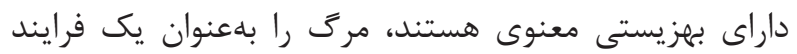

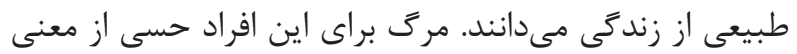

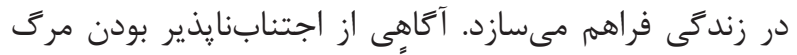

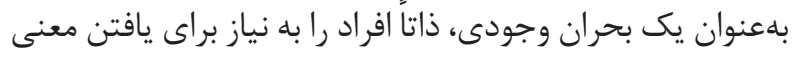

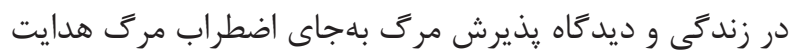

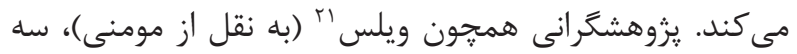

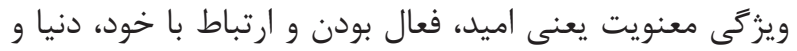

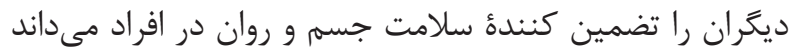

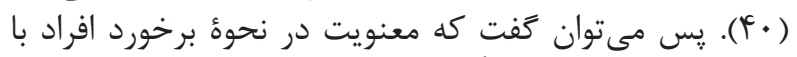

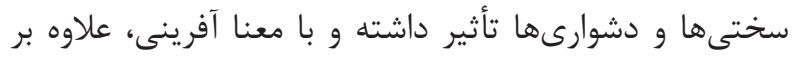

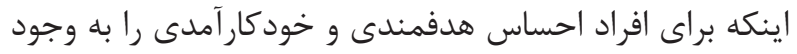

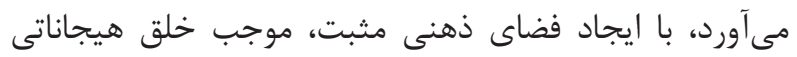

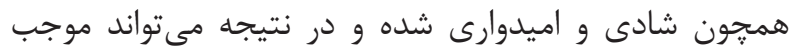
كاهش اضطراب مرك در سالمندان كردد.

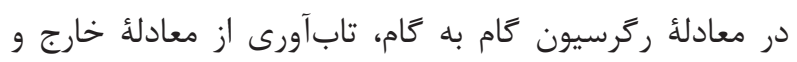

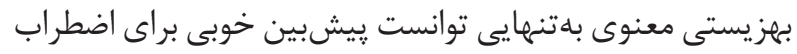

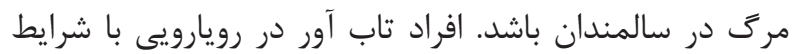

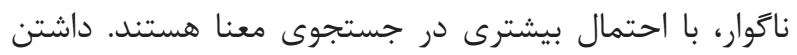

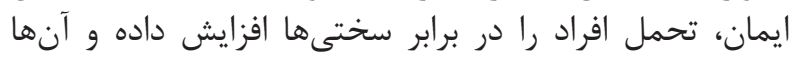

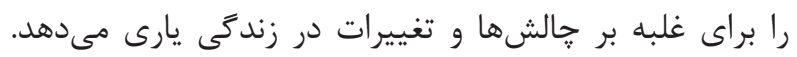

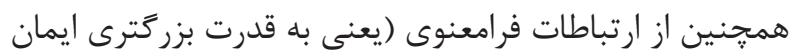

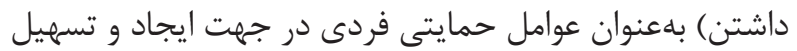

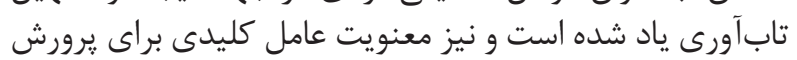

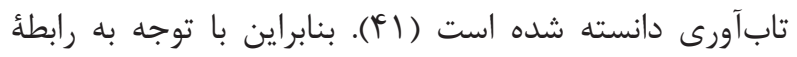

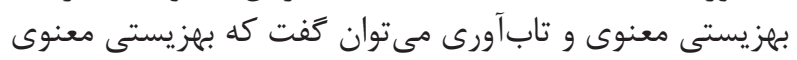

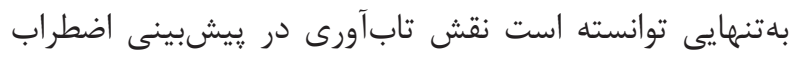

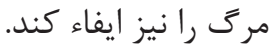

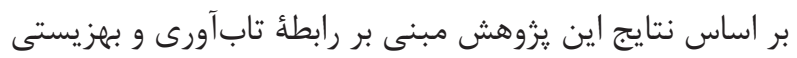

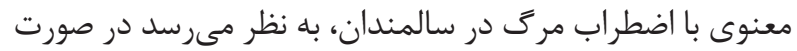

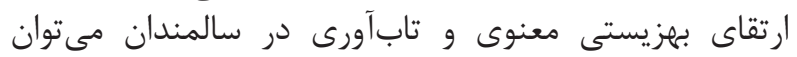

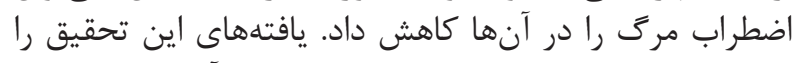

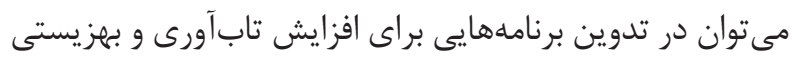
معنوى در سالمندان به كار برد.

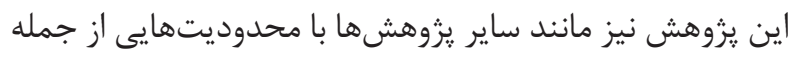

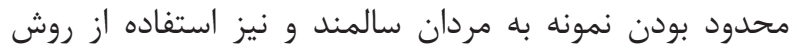

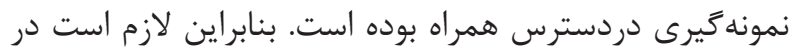

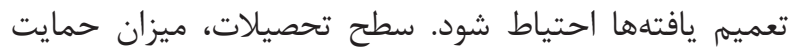

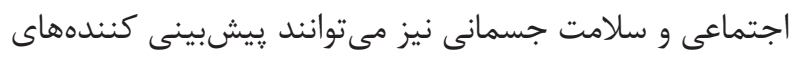

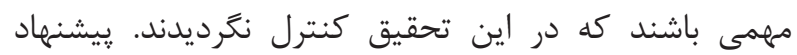
مىشود در يزوهشهاى آتى بهعنوان موضوعى مستقل به به آنها

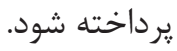

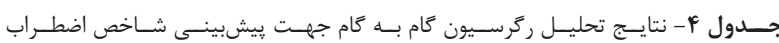
مركى از روى متغيرهاى بهزيستى معنوى.

\begin{tabular}{|c|c|c|c|c|c|c|}
\hline \multirow{2}{*}{ فريب تعيين (R2) } & \multirow{2}{*}{ هنى إنى } & \multirow{2}{*}{ آمارةرً } & \multirow{2}{*}{ فرايب استأدارن } & \multicolumn{2}{|c|}{ فرايب غير استاندارن } & \multirow{2}{*}{ ثناخصنهائ مدل } \\
\hline & & & & أنحراف ميعيار & B & \\
\hline \multirow{2}{*}{.194} & $\%$ & $91, Y 0$ & - & $\pi \%$ & rerA & ضريب ثابت \\
\hline & $\cdot 1$ & $-Y 1 / 99$ & $-1 / 19$ & 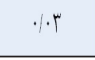 & -191 & بيزيسنى مثنوى \\
\hline
\end{tabular}

بحث و نتيجه گيرى

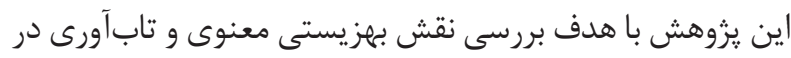

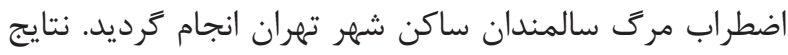

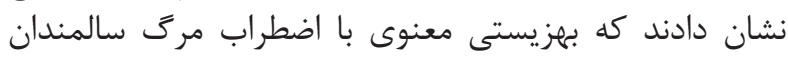

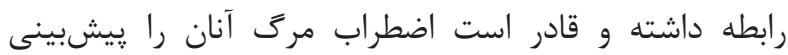

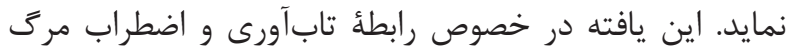

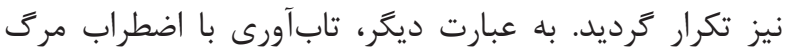

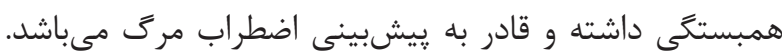

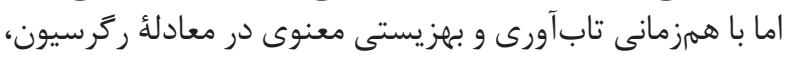

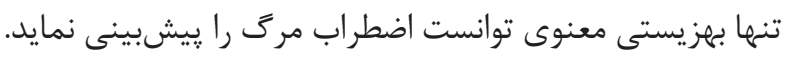

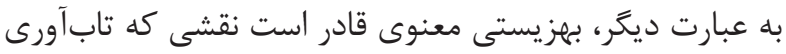

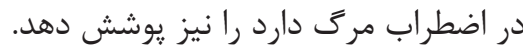

نتايج اين يزوهش در خصوص رابطؤ بهزيستى معنوى و اضطراب

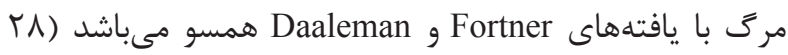

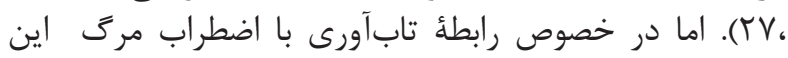

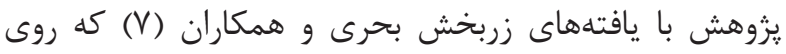

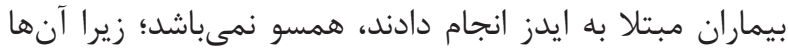

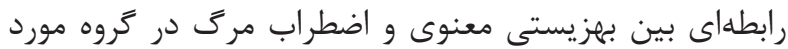

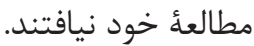

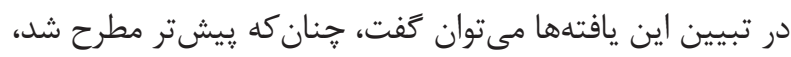

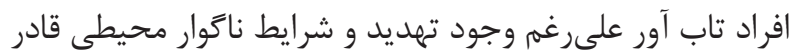

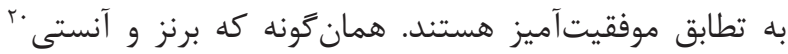

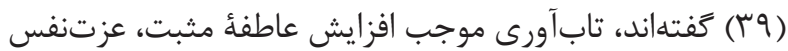

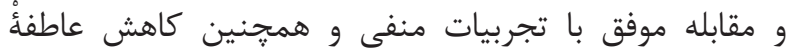

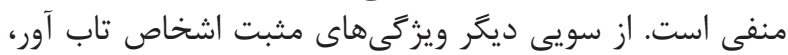

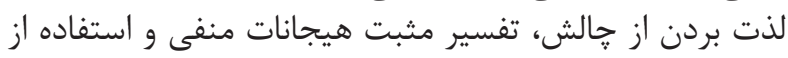

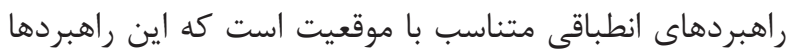

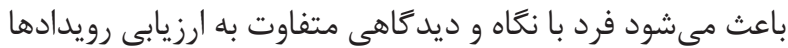

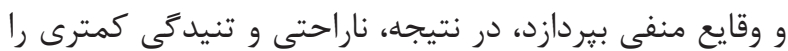
تجربه كند و بهتر با آن رويداد كنار آيد.

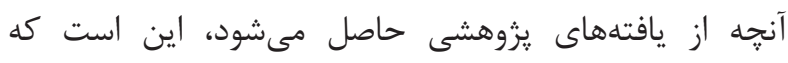

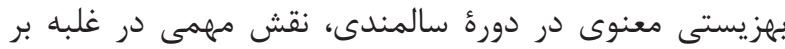

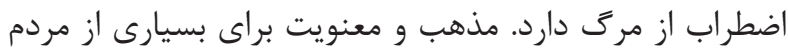

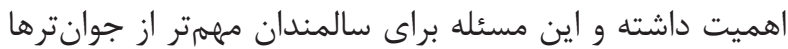

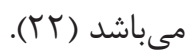


1. Naderi F, Roushani KH. Relation between spiritual intelligence, social intelligence and death anxiety in Ahwaz woman senile. Woman Cult. 2011; 2(6): 55-67.

2. Imanifar H, Bostani G, Dodman F, Raiesi R. Facing with death from Quran and psychology viewpoints. J Interdic Res Quran. 2011; 2(4): 65-72.

3. Naderi F, Shokouhi M. Relationship of optimism, humor and social maturity with death anxiety in Ahvaz Golestan hospital nurses. J New Find Psychol. 2009; 4(10): 85-94.

4. Berk LE. Development through the lifespan. $4^{\text {nd }}$ ed. Tehran: arasbaran publishers. 2001; p. 476.

5. Ghasempour A, Sooreh J, Seid tazeh kand MT. Predicting death anxiety on the basis of emotion cognitive regulation strategies. Knowl Res Appl Psychol. 2012; 13(2): 63-70.

6. Singh C. An Analytic Study of Death Anxiety among Type 2 Diabetes. Mediterr J Soc Sci. 2013; 4 (11): 205-17.

7. Zarbakhsh Bahri MR, Pourhassani SA, Rahmani M, Zalekan F, Kianipour E. The relationship between life expectancy and resilience with Death anxiety in HIV patients. J Basic Appl Sci Res. 2013; 3(6): 899-904.

8. Hui VK, Fung HH. Mortality anxiety as a function of intrinsic religiosity and perceived purpose in life. Death Stud. 2008; 33(1): 30-50.

9. Gomez R, Fisher JW. Domains of spiritual well-being and development and validation of the Spiritual WellBeing Questionnaire. Pers Individ Dif. 2003; 35: 1975-91.

10. Jafari E, Hajloo N, Faghani R, Khazan K. The relationship between spiritual well-being, hardiness, and mental health in the elderly. J Res in Behav Sci. 2012; 10(6): 431-40.

11. Hosseini Vajari K, Bahrami Ehsan H. The role of religious coping and spiritual well being in explanation of mental health. J Psychol. 2005; 9(3): 248-60.

12. Martinez BB, Custodio RP. Relationship between mental health and spiritual wellbeing among hemodialysis patients: a correlation study. Sao Paulo Med J. 2014; 132(1): 7-23.

13. Jafari E, Dehshiri GH, Eskandari H, Najafi M, Heshmati R. Spiritual well-being and mental health in university students. Procedia-Soc Beh Sci. 2010: 1477-81.

14. Lanfredi M, Candini V, Buizza C, Ferrari C, Boero ME, Giobbio GM, et al. The effect of service satisfaction and spiritual well-being on the quality of life of patients with schizophrenia. Psychiatry Res. 2014; 216(2): 185-91.

15. Bickerton GR, Miner MH, Dowson M, Griffin B. Spiritual resources as antecedents of clergy well-being. J Vocat Behav. 2015; 87: 123-33.

16. Ghobari Bonab B, Motavalipoor A, Habibi Asgarabadi M. Relationship between anxiety and depression and magnitude of spirituality in students of the University of Tehran. J Apple Psychol. 2009; 3(2): 110-23.

17. Safaee Rad I, Karimi L, Shomoossi N, Ahmadi tahor M. The relationship between spiritual well being and mental health of university students. J Sabzevar Uni Med Sci. 2011; 17(4): 274-80.

18. Genia V. Evaluation of spiritual well-being scale in sample of college student. Int J Psychol Relig. 2001; 11(1): 25-30.

19. Kim J, Heinemann AW, Bode RK, Sliwa J, King RB. Spirituality, quality of life, and functional recovery after medical rehabilitation. Rehabil Psychol. 2000; 45(4): 365-85.

20. Vallurupalli M, Lauderdale K, Balboni MJ, Phelps AC, Block SD, Ng AK, et al. The role of spirituality and religious coping in the quality of life of patients with advanced cancer receiving palliative radiation therapy. $\mathrm{J}$ Support Oncol. 2012; 10(2): 81-7.

21. Jafari N, Farajzadegan Z, Loghmani A, Majlesi M, Jafari N. Spiritual Well-being and Quality of life of Iranian adults with type 2 diabets. Evid Based Complement Alternat Med. 2014; doi: 10.1155/2014/619028.

22. Koenig HG. Spirituality, wellness, and quality of life. Sex Reprod Menopause. 2004; 2(2): 76-82.

23. Motamedi A, Ezhei J, Azad Fallah P, Keiamanesh A. The relationship between religious trends and successful aging. Train Learn Res. 2005; 10(12): 43-56.

24. Ali Akbari Dehkordi M, Oraki M, Barghi Irani Z. Relation between religious orientation with anxiety about death, and alienation in aged peoples (seniors) in Tehran. Soc Psychol Res. 2011; 1(2): 140-59. 


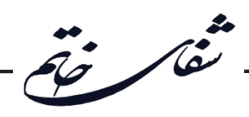

25. Ardelt M. Effects of religion and purpose in life on elders' subjective well-being and attitudes toward death. J Relig Gerontol. 2003; 14(4): 55-65.

26. Hadzic M. Spirituality and mental health: current research and future directions. J Spiritual Ment Health. 2011; 13: 223-35.

27. Fortner BV, Neimeyer RA. Death anxiety in older adults: A quantitative review. Death Stud. 1999; 23(5): 387-411.

28. Daaleman TP, Dobbs D. Religiosity, spirituality and death attitudes in chronically III older adults. Res Aging. 2010; 32(2): 224-43.

29. Newman R. APA's resilience initiative. Professional psychology. Res Pract. 2005; 36(3): 227-9.

30. Fernando M, Chowdhury R. The relationship between spiritual well-being and ethical orientations in decision making: an empirical study with business executives in Australia. J Bus Ethics. 2010; 95 (2): 211-25.

31. Smith L, Webber R, DeFrain J. Spiritual wellbeing and its relationship to resilience in young people: A mixed methods case study. 2013: 1-13. doi: $10.1177 / 2158244013485582$.

32. Hagh Ranjbar F, Kakavand A, Borjali A, Bermas H. Resiliency and quality of life in mothers of children with intellectual disability. J Health Psychol. 2011; 1(1): 179-89.

33. Besharat MA. Resilience, vulnerability \& mental health. J Psychol Sci. 2008; 24: 373-83.

34. Deblinger E, Runyon MK, Steer RA. Profiles of personal resiliency in youth who have experienced physical or sexual abuse. J Psychoeduc Assess. 2014; 32(6): 558-66.

35. Baldwin DR, Jackson D, Okoh I, Cannon RL. Resiliency and Optimism: an African American senior citizens perspective. J Black Psychol. 2011; 37(1): 24-41.

36. Dehshiri Gh, Sohrabi F, Jafari E, Najafi M. Investigation of psychometric properties of spiritual well-being scale among students. Psychol Stud. 2009; 4(3): $35-45$.

37. Samani S, Jokar B, Sahragard N. Effects of resilience on mental health and life satisfaction. Iran J Psychiatry Clin Psychol. 2007; 13(3): 290-5.

38. Tavakoli MA, Ahmadzadeh B. Investigation of validity and reliability of Templer death anxiety scale. IJPCP. 2011; 6(21): 72-80.

39. Burns RA, Anstey JK. The Connor-Davidson Resilience Scale (CD-RISC): Testing the invariance of a unidimensional resilience measure that is independent of positive and negative affect. Pers Individ Dif. 2010; 48: 527-31.

40. Momeni K, Shahbazi rad A. Relationship of spirituality, resiliency, and coping strategies with quality of life in university students. J Behav Sci. 2012; 6(2): 626-34.

41. Hosseini Ghomi T, Salami Bajestani H. The relationship between religious orientation and resiliency among mothers of children with diseases. RavanshenasiVa-Din. 2011; 4(3): 69-82. 